\title{
Study on Dynamic Response of Downhole Tools under Perforation Impact Load
}

\author{
Liu Jun, ${ }^{1}$ Li Shide, ${ }^{1}$ Liu Qingyou, ${ }^{1,2}$ Liu Xian, ${ }^{1}$ Guo Xiaoqiang, ${ }^{1}$ and He Yufa ${ }^{3}$ \\ ${ }^{1}$ School of Mechatronic Engineering, Southwest Petroleum University, Chengdu, Sichuan 610500, China \\ ${ }^{2}$ Xihua University, Chengdu, Sichuan 610039, China \\ ${ }^{3}$ CNOOC Research Institute, Beijing 100028, China
}

Correspondence should be addressed to Liu Qingyou; liuqy66@aliyun.com

Received 22 March 2017; Revised 22 June 2017; Accepted 12 July 2017; Published 24 August 2017

Academic Editor: Miguel Neves

Copyright (C) 2017 Liu Jun et al. This is an open access article distributed under the Creative Commons Attribution License, which permits unrestricted use, distribution, and reproduction in any medium, provided the original work is properly cited.

\begin{abstract}
A model of a multibody system is established to investigate the dynamic response of an oil tube-shock absorber-perforating gun system in downhole perforation-test joint operation. In the model, the oil tube and perforating gun are modeled as elastic rods and the shock absorber is modeled as single particle system with damping and a spring. Two force continuity conditions are used to simulate the interactions among the three components. The perforation impact load is determined by an experiment of underwater explosion of perforating bullets. Using the model, the effects of charge quantity of perforating bullet, the number of shock absorbers, and the length of oil tube on the dynamic response of oil tube and packer are investigated. On this basis, a basic principle of the combination design of shock absorber and oil tube is proposed to improve the mechanical state of downhole tools. The study results can provide theoretical support for the design of downhole perforation-test joint operation.
\end{abstract}

\section{Introduction}

In the perforation-test joint operation of oil-gas development, in order to improve perforation efficiency, high perforation density and perforating bullet with high explosive charge are widely used, resulting in the strength increase of explosive load as well as worse mechanical state of the downhole tools, as shown in Figure 1. In extreme conditions, the explosive load may cause overall buckling of tube string and damage of downhole tools $[1,2]$ including test equipment and packer, as shown in Figure 2. So, how to deal with the contradiction between the improvement of perforation efficiency and the safety of the tube string system becomes an urgent problem to be solved.

The study on the mechanics of tube string can be traced back to the helical buckling theory of packer pipe string, which was put forward by Lubinski et al. [3] in 1962. However, it is only in recent years that the research on the mechanics of the perforated tube string has been carried out [4-11]. Using the gap element theory of finite element method (FEM), a three-dimensional static analysis model of a perforated tube string was established by Zhang et al. [5]. Yin et al. [12] also used a static analysis model to examine the effect of high pressure and perforation gun on tube string and showed that the high pressure formed in the sealing section at the moment of perforation is one of the main factors that cause the vibration of the string and the damage of test equipment.

A cross combination scheme of shock absorber and damping oil tube was proposed by Fan and Li [13] to minimize the effects of the shock wave energy on packer and test instrument. However, this method lacks theoretical basis. A ground simulation test system has been used by Lu et al. [14] to investigate the pressure and acceleration responses at the end of a perforation tube string. A preliminary understanding of the dynamic response characteristics of perforation string suffering explosion shock load is obtained. However, the dynamic response of under tube string and the effect of impact load on packer remained unclear.

A vibration model of perforated tube-shock absorber was established by Liu et al. [15] to investigate the influences of system parameters on the vibration response of oil tube. However, in the model the effect of perforation gun is not considered and the perforating load was not verified by experimental data. 


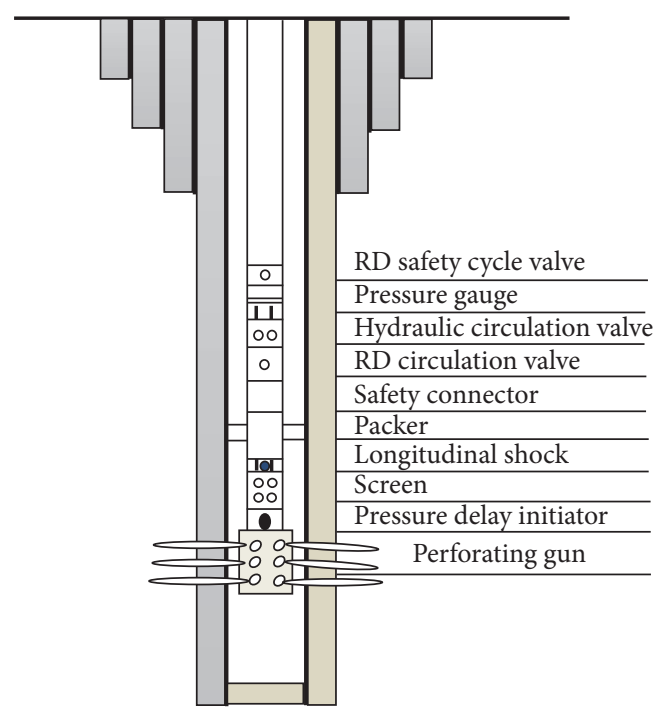

FIGURE 1: Downhole tools in perforation-test joint operation.

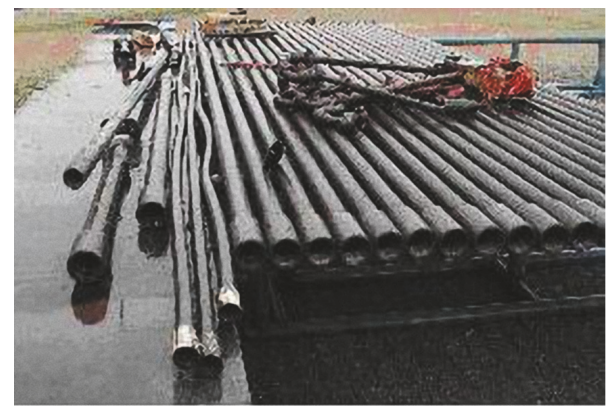

FIGURE 2: Tube buckling caused by perforation load.

Zhou [16], Teng [17], and Kang et al. [18] applied the software of LS-DYNA to simulate the process of perforation bullet explosion, jet formation, and the jet penetration of casingcement- stratum. Zhang et al. [19] used ANSYS/AUTODYN software to investigate the effects of the length of perforation zone, the interval time of perforation bullet explosive, the charge quantity in a perforation bullet, the physical conditions of artificial bottom hole, "pocket" depth, and other parameters on the perforation impact load. Li et al. [20] presented a dimensionless expression of perforation impact loads as well as its engineering calculation formula. Based on the formula, the buckling behavior of the perforation tube string of an actual well was analyzed. In these studies, the focuses were put on explosive process of perforating bullet and the pressure distribution in the wellbore. The effects of perforation parameters on the dynamic response and stability of oil tube and the force acting on packer remained unclear.

A longitudinal vibration model coupling the testing tube and shock absorber was used by Huang [21] to investigate the effects of perforation parameters on the dynamic behaviors of oil tube and packer. Another dynamic model for oil tubes in curved wells was also proposed by Cai et al. [22] to analyze the radial displacement, contact force, friction resistance, and axial force in oil tube. In the model, the nonlinear contact

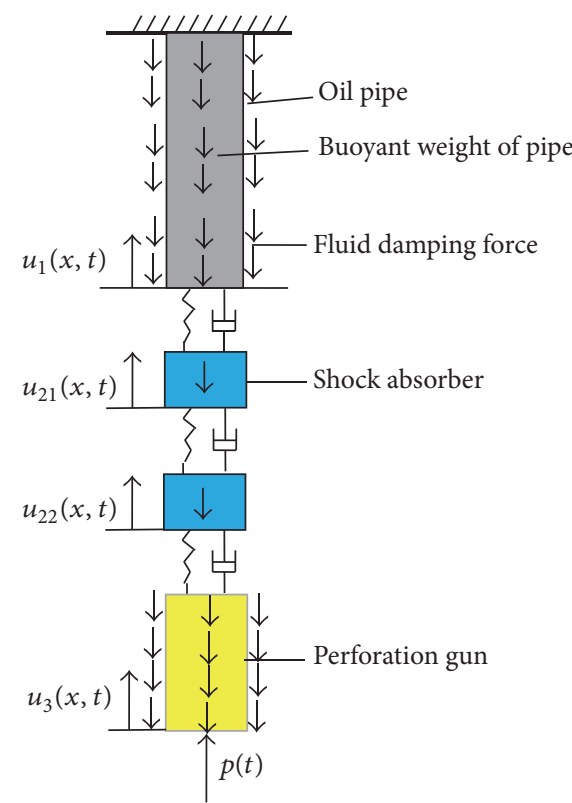

FIGURE 3: Mechanical model of oil tube-shock absorber-perforating gun.

between oil tube and casing is simulated by the space beam element and spring element. In these studies, the mutual coupling effect of oil tube, shock absorber, and perforation gun and the effect of perforation parameters on the stability of oil tube and the force acting on packer were not considered. Effective experimental data are still lacking.

The purpose of this paper is to find an effective engineering calculation formula of shock load based on experiment data and establish a dynamic model considering the mutual coupling effect of oil tube, shock absorber, and perforation gun. On this basis, the effects of main perforation parameters on the dynamic response of downhole tools are studied, focusing on the buckling of oil tube and the force acting on packer.

\section{Vibration Differential Equation of Downhole Tools}

2.1. Basic Assumptions and Mechanical Model. According to the structure of the perforation string system and the operation condition, the following assumptions are made in order to derive the dynamic governing equations of the system.

(1) It is assumed that the material of oil tube and perforation gun is homogeneous and isotropic.

(2) The shock absorber is equivalent to a mass-springdamping system, ignoring the geometry of the shock absorber and the inhomogeneity of the mass distribution.

(3) The packer is considered as a fixed support without considering the relative displacement between the packer and casing tube or oil tube.

(4) The structural damping of the tube string is ignored.

(5) Only the longitudinal vibration of the tube string is considered.

On the basis of these assumptions, the mechanical model of downhole tools can be described in Figure 3. 


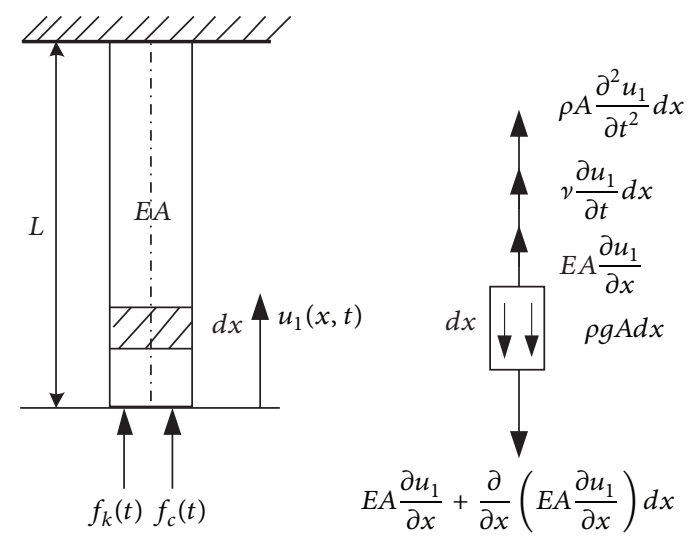

FIgUre 4: Mechanical analysis of an oil tube element.

2.2. Longitudinal Vibration Differential Equation of a Downhole Tube. The oil tube is taken as an example to illustrate the process of establishing the vibration differential equation of a tube string including oil tube and perforation gun. The coordinate system and the mechanical analysis of an oil tube element are shown in Figure 4. The forces on the element are composed of inertia force $\rho A\left(\partial^{2} u_{1} / \partial t^{2}\right) \mathrm{d} x$, the damping force of the liquid in annular $\nu\left(\partial u_{1} / \partial t\right) \mathrm{d} x$, gravity $\rho g A \mathrm{~d} x$, and the elastic force in the tube $E A \partial u_{1} / \partial x$, where $E$ is elastic modulus, $A$ is the cross section area of the oil tube, $\rho$ is density, $v$ is the damping coefficient of the liquid in annulus, and $g$ is gravity acceleration.

The equilibrium equation of the element is given by

$$
\begin{aligned}
& \rho A \frac{\partial^{2} u_{1}}{\partial t^{2}} \mathrm{~d} x+E A \frac{\partial u_{1}}{\partial x}+\nu \frac{\partial u_{1}}{\partial t} \mathrm{~d} x \\
& -\left(E A \frac{\partial u_{1}}{\partial x}+\frac{\partial}{\partial x}\left(E A \frac{\partial u_{1}}{\partial x}\right) \mathrm{d} x\right)-\rho g A \mathrm{~d} x=0 .
\end{aligned}
$$

Based on the transformation of (1), the partial differential equation of the vibration of an oil tube string can be expressed as

$$
\frac{\partial^{2} u_{1}}{\partial t^{2}}-a^{2} \frac{\partial^{2} u_{1}}{\partial x^{2}}+v_{0} \frac{\partial u_{1}}{\partial t}=g
$$

where $a=\sqrt{E / \rho}$ is the propagation speed of stress wave in perforation tube string; $v_{0}$ is the damping coefficient of liquid inside and outside the oil tubing. A specific expression of $v_{0}$ is given in [23]

$$
\begin{aligned}
v_{0} & =\frac{12 \pi \mu}{\rho A}\left(\frac{D_{r}}{D_{t i}-D_{r}}\right)\left[\left(0.20+0.39 \frac{D_{r}}{D_{t i}}\right)\right. \\
& \left.+\frac{2.1970 \times 10^{4}}{24}\left(\frac{D_{c}}{D_{t i}}-0.3810\right)^{2.57} \frac{D_{c}^{2}-D_{r}^{2}}{L D_{r}}\right],
\end{aligned}
$$

where $\mu$ is the dynamic viscosity of the fluid, $D_{c}$ is the external diameter of oil tube, $D_{t i}$ is internal diameter, and $D_{r}$ is the diameter of borehole.

The local coordinates and the force analysis of the shock absorber are shown in Figure 5, where $f_{k 1}$ and $f_{c 1}$ are, respectively, the spring and damping forces between the shock absorber and oil tube, $f_{k 2}$ and $f_{c 2}$ are correspondent forces between the shock absorber and perforation gun, and $f_{I 2}$ and $m_{2} g$ are, respectively, the inertia force and gravity of the shock absorber.

The equilibrium equation of the shock absorber is given as

$$
f_{k 1}+f_{c 1}=m_{2} g+f_{I 2}+f_{k 2}+f_{c 2} \text {. }
$$

Equation (4) can be further extended to

$$
\begin{gathered}
c \frac{d}{d t}\left[u_{1 d}(t)-u_{2}(t)\right]+k\left[u_{1 d}(t)-u_{2}(t)\right] \\
=m_{2} \frac{d^{2} u_{2}}{d t^{2}}+c \frac{d}{d t}\left[u_{2}(t)-u_{3 u}(t)\right] \\
+k\left[u_{2}(t)-u_{3 u}(t)\right]+m_{2} g
\end{gathered}
$$

where $u_{1 d}(t), u_{2}(t)$, and $u_{3 u}(t)$ are, respectively, the displacements of the lower end of the oil tube, shock absorber, and the upper end of perforating gun. $m_{2}, k$, and $c$ are, respectively, the mass, stiffness coefficient, and damping coefficient of the shock absorber.

2.3. Solving Scheme. Using Newton's center difference formula to discretize (2) and (5), the following numerical forms are given, respectively, as (6) and (7).

Numerical form of the longitudinal vibration equation of oil tube and perforation gun:

$$
u_{i, j+1}=\frac{a^{2} \Delta t^{2} / \Delta x^{2}\left(u_{i+1, j}+u_{i-1, j}\right)-\left(2\left(a^{2} \Delta t^{2} / \Delta x^{2}\right)-2.0-v_{0} \Delta t\right) u_{i, j}-u_{i, j-1}+g \Delta t^{2}}{1+v \Delta t} \quad 1 \leq i \leq N-1,1 \leq j \leq K .
$$

Numerical form of the vibration equation of a shock absorber:

$$
\begin{gathered}
c\left(\frac{u_{1, j+1}-u_{1, j}}{\Delta t}-\frac{u_{i+2, j+1}-u_{i+2, j}}{\Delta t}\right) \\
+k\left(u_{1, j+1}-u_{i+2, j+1}\right)
\end{gathered}
$$

$$
\begin{aligned}
= & \frac{m_{2}}{\Delta t^{2}}\left(u_{i+2, j+1}-2 u_{i+2, j}+u_{i+2, j-1}\right) \\
& +c\left(\frac{u_{i+2, j+1}-u_{i+2, j}}{\Delta t}-\frac{u_{i+3, j+1}-u_{i+3, j}}{\Delta t}\right) \\
& +k\left(u_{i+2, j+1}-u_{i+3, j+1}\right)+m_{2} g .
\end{aligned}
$$




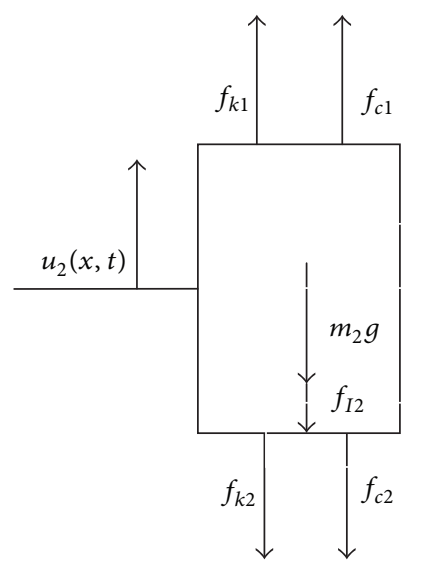

FIGURE 5: Force analysis of a shock absorber.

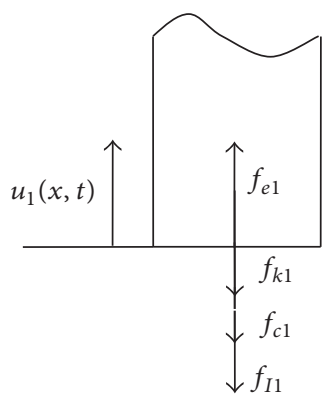

FIGURE 6: Force analysis of the lower end of oil tube.

Let $x_{2}=c / \Delta t, x_{6}=m_{2} / \Delta t^{2} ;(7)$ can be written as

$$
\begin{aligned}
\left(x_{2}+\right. & k) u_{1, j+1}+\left(-2 x_{2}-2 k-x_{6}\right) u_{i+2, j+1} \\
& +\left(x_{2}+k\right) u_{i+3, j+1} \\
= & x_{2} u_{1, j}-x_{2} u_{i+2, j}-2 x_{6} u_{i+2, j}+x_{6} u_{i+2, j-1} \\
& -x_{2} u_{i+2, j}+x_{2} u_{i+3, j}+m_{2} g .
\end{aligned}
$$

By solving (6), the nodal displacements of a perforation tube string system at moment $j+1$ can be obtained, except for those of the lower end of oil tube, shock absorber, and the upper end of perforating gun. To determine the three unknown displacements, besides (8), at least two additional equations are needed. Here, two force continuity conditions are introduced.

The force analysis of the lower end of the oil tube is shown in Figure 6. According to the law of force continuity, the balance equation of these forces is given by

$$
\left.E A \frac{\partial u_{1}}{\partial x}\right|_{x=L}=f_{c 1}+f_{k 1}+f_{I 1}
$$

where $f_{I 1}$ is the inertia force of the lower element of oil tube.

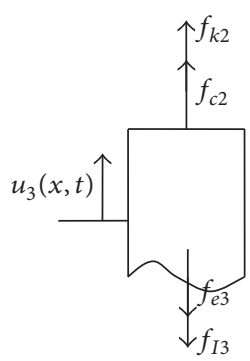

FIGURE 7: Force analysis of the upper end of perforation gun.

Using Newton's center difference formula, the numerical form of (9) can be expressed as

$$
\begin{aligned}
E_{O} A_{O} & \frac{u_{2, j+1}-u_{1, j+1}}{l_{o e}} \\
= & c \frac{u_{1, j+1}-u_{1, j}-u_{i+2, j+1}+u_{i+2, j}}{\Delta t} \\
& +k\left(u_{1, j+1}-u_{i+2, j+1}\right) \\
& +m_{o e} \frac{u_{1, j+1}-2 u_{1, j}+u_{1, j-1}}{\Delta t^{2}} .
\end{aligned}
$$

The oil tube is discretized by a finite number of elements. So, here $m_{o e}$ denotes the mass of the bottom element of the oil tube and can be expressed as $m_{o e}=1 / 4 \pi\left(D_{c}^{2}-D_{t i}^{2}\right) l_{o e} \rho$, where $l_{o e}$ is the element length. Let $x_{1}=E_{o} A_{o} / l_{o e}, x_{3}=m_{o e} / \Delta t^{2}$; (10) can be written as

$$
\begin{aligned}
& \left(-x_{1}-x_{2}-k-x_{3}\right) u_{1, j+1}+\left(x_{2}+k\right) u_{i+2, j+1} \\
& =-x_{1} u_{2, j+1}-x_{2} u_{1, j}+x_{2} u_{i+2, j}-2 x_{3} u_{1, j} \\
& \quad+x_{3} u_{1, j-1} .
\end{aligned}
$$

The force analysis of the upper end of a perforation gun is shown in Figure 7. The balance equation of these equations is given by

$$
f_{c 2}+f_{k 2}=\left.E_{p} A_{p} \frac{\partial u_{3}}{\partial x}\right|_{x=0}+f_{I 3} .
$$

Using Newton's center difference formula, the numerical form of (12) can then be written as

$$
\begin{aligned}
k\left(u_{i+2, j+1}-u_{i+3, j+1}\right) & \\
& +\frac{c}{\Delta t}\left(u_{i+2, j+1}-u_{i+2, j}-u_{i+3, j+1}+u_{i+3, j}\right) \\
= & \frac{E_{p} A_{p}}{l_{p e}}\left(u_{i+3, j+1}-u_{i+4, j+1}\right) \\
& +\frac{m_{e p}}{\Delta t^{2}}\left(u_{i+3, j+1}-2 u_{i+3, j}+u_{i+3, j-1}\right) .
\end{aligned}
$$

The performed gun is also discretized by a finite number of elements. So, here $m_{p e}$ denotes the mass of the top element 
TABLE 1: Experimental site and equipment.

\begin{tabular}{ll}
\hline Experimental site & A pool with diameter $2.0 \mathrm{~m}$ and water depth $2.7 \mathrm{~m}$ \\
\hline \multirow{2}{*}{ Instruments } & 138A51 and 138A26 underwater explosion pressure sensor, F482A51 constant current source, \\
& $\begin{array}{l}\text { DPO4034 Tektronix storage oscilloscope, computer, blasting line, data transmission line, } \\
\text { detonator, trigger mutual inductor, positioning bracket. }\end{array}$ \\
\hline Test material & $\begin{array}{l}\text { Primer detonator, detonating cord, perforating bullet, perforating gun, contrast HMX bare drug } \\
\text { column. }\end{array}$ \\
\hline
\end{tabular}

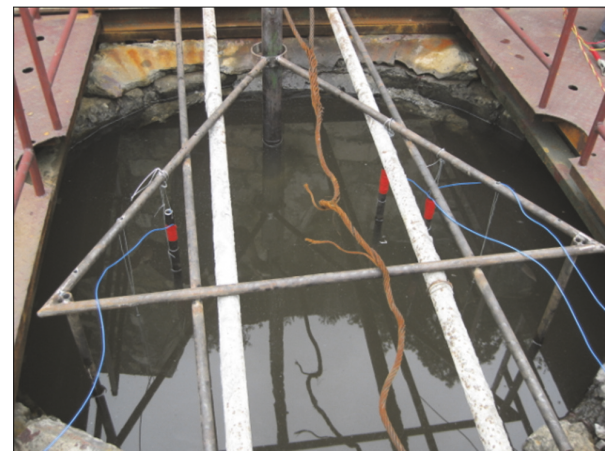

FIGURE 8: Explosion test pool and positioning bracket.

of the performed gun and can be written as $m_{p e}=1 / 4 \pi\left(D_{p c}^{2}{ }^{-}\right.$ $\left.D_{p i}^{2}\right) l_{p e} \rho$, where $D_{p c}, D_{p i}$, respectively, denote the external diameter and internal diameter of perforation gun; $l_{p e}$ is the element length. Let $x_{4}=E_{p} A_{p} / l_{p e}, x_{5}=m_{p e} / \Delta t^{2} ;(13)$ can be written as

$$
\begin{aligned}
& \left(k+x_{2}\right) u_{i+2, j+1}+\left(-k-x_{2}-x_{4}-x_{5}\right) u_{i+3, j+1} \\
& =x_{2} u_{i+2, j}-x_{2} u_{i+3, j}-x_{4} u_{i+4, j+1}-2 x_{5} u_{i+3, j} \\
& \quad+x_{5} u_{i+3, j-1} .
\end{aligned}
$$

Solving (8), (11), and (14) simultaneously, the displacements of the lower end of oil tube, shock absorber, and the upper end of perforating gun at moments $j+1, u_{1, j+1}, u_{i+2, j+1}$, and $u_{i+3, j+1}$ can be determined.

\section{Experimental Analysis of Downhole Perforation Load}

An underwater explosion experiment of perforation bullet was carried out to investigate the pressure distribution of perforation explosion. The equipment needed for the experiment is mainly composed of a water tank, perforation guns, ammunition, and sensors which are shown in Table 1 and Figures 8 and 9 .

In the experiment, the perforation gun with perforation bullet is placed in the pool, and the explosion pressure sensors are arranged in the position shown in Figure 10. After the perforation bullet is detonated, the shock wave energy, namely, equivalent energy (as shown in Figure 11), can be collected by the pressure sensors, based on which the quality of equivalent explosive can be determined.

More than twenty underwater explosion tests on 5 kinds of high temperature and high pressure oil perforation bullet

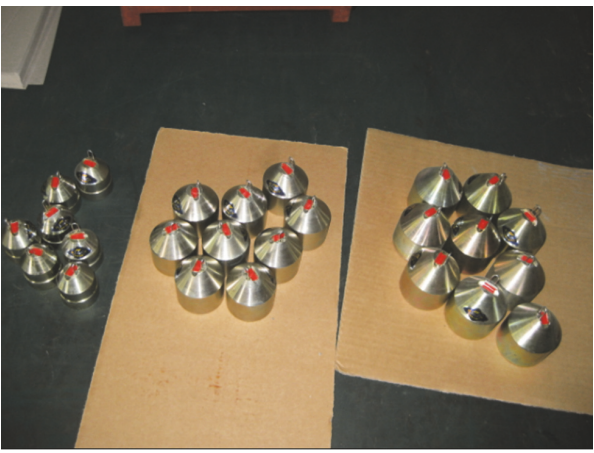

FiguRE 9: Perforating bullet for explosive test.

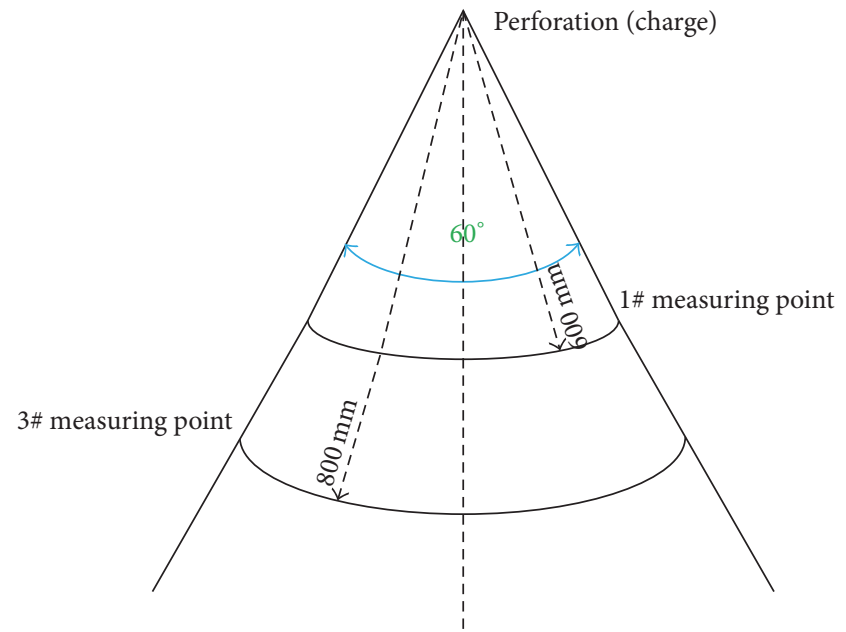

FIGURE 10: Arrangement diagram of pressure sensors.

were carried out to measure the underwater explosion overpressure which is shown in Table 2.

Based on the tested data in the experiment, the mass percentages $(Q)$ of explosive mass to total charge mass $(M)$ are determined. Here, explosive mass refers to those consumed by the shock wave produced by underwater explosion and the bubble pulsation of perforator tapes 73, 89, and 102 (main blasting charge is HMX). Figure 12 indicates that the mass percentage increases with increasing the charge quantity of perforation gun.

With linear fitting the experiment data of types 73,89 , and 102 , an equation describing the relation between the mass percentage and total charge mass is obtained.

$$
Q=0.31624+0.00181 M .
$$


TABLE 2: Explosion overpressure values of perforation bullet measured in the experiment.

\begin{tabular}{|c|c|c|c|c|c|c|c|c|}
\hline \multirow{2}{*}{ Serial number } & \multirow{2}{*}{\multicolumn{2}{|c|}{ Types of perforation bullet }} & \multicolumn{6}{|c|}{ Explosion overpressure $(\mathrm{MPa})$} \\
\hline & & & \multicolumn{2}{|c|}{$1^{\#}$ sensor $(0.6 \mathrm{~m})$} & \multicolumn{2}{|c|}{$2^{\#}$ sensor $(0.8 \mathrm{~m})$} & \multicolumn{2}{|c|}{$3^{\#}$ sensor $(0.8 \mathrm{~m})$} \\
\hline 1 & \multirow{4}{*}{$\begin{array}{c}\text { Type } 73(18 \mathrm{~g} \\
\text { HMX) } \\
\text { with } 73 \mathrm{~mm} \\
\text { perforating gun }\end{array}$} & \multirow{3}{*}{1 perforation bullet } & 15.181 & \multirow{3}{*}{15.460} & 12.102 & & 12.013 & \multirow{3}{*}{11.871} \\
\hline 2 & & & 16.017 & & 10.774 & 11.747 & 11.73 & \\
\hline 3 & & & \multirow{2}{*}{\multicolumn{2}{|c|}{15.181}} & \multicolumn{2}{|l|}{12.364} & \multirow[t]{2}{*}{-} & \\
\hline 4 & & 3 perforation bullets & & & \multicolumn{2}{|c|}{14.543} & & 14.358 \\
\hline 5 & \multirow{4}{*}{$\begin{array}{c}\text { Type } 89(25 \mathrm{~g} \\
\text { HMX) } \\
\text { with } 89 \mathrm{~mm} \\
\text { perforating gun }\end{array}$} & \multirow{3}{*}{1 perforation bullet } & 17.967 & \multirow{3}{*}{17.943} & 13.427 & & 12.144 & \multirow{3}{*}{13.107} \\
\hline 6 & & & 18.245 & & 13.017 & 12.952 & 13.7 & \\
\hline 7 & & & 17.618 & & \multicolumn{2}{|l|}{12.412} & \multirow{2}{*}{\multicolumn{2}{|c|}{$\begin{array}{r}13.478 \\
17.256\end{array}$}} \\
\hline 8 & & 3 perforation bullets & & & & & & \\
\hline 9 & \multirow{4}{*}{$\begin{array}{l}\text { Type } 102(32 \mathrm{~g} \\
\text { HMX) } \\
\text { with } 102 \mathrm{~mm} \\
\text { perforating gun }\end{array}$} & \multirow{3}{*}{1 perforation bullet } & 19.359 & \multirow{3}{*}{21.588} & 15.688 & & - & \multirow{3}{*}{15.862} \\
\hline 10 & & & & & 15.458 & 15.786 & 16.216 & \\
\hline 11 & & & 22.423 & & \multicolumn{2}{|l|}{16.212} & \multirow{2}{*}{\multicolumn{2}{|c|}{15.508}} \\
\hline 12 & & 3 perforation bullets & & & & & & \\
\hline 17 & \multirow{4}{*}{$\begin{array}{c}\text { Type } 89 \text { (23g PYX) } \\
\text { with } 89 \mathrm{~mm} \\
\text { perforating gun }\end{array}$} & \multirow{3}{*}{1 perforation bullet } & 16.365 & \multirow{3}{*}{16.481} & 11.102 & & 12.144 & \multirow{4}{*}{12.40} \\
\hline 18 & & & 15.808 & & - & 11.462 & 11.366 & \\
\hline 19 & & & 17.27 & & 11.822 & & 13.7 & \\
\hline 20 & & 3 perforation bullets & & & & & 15.559 & \\
\hline 21 & Type 102 (31 g & & 22.981 & & - & & 14.256 & \\
\hline 22 & PYX) & 1 perforation bullet & 19.359 & 21.077 & 14.458 & 14.40 & 15.7 & 14.7 \\
\hline 23 & with $102 \mathrm{~mm}$ & & 20.891 & & 14.343 & & 14.144 & \\
\hline 24 & perforating gun & 3 perforation bullets & & & & & & \\
\hline
\end{tabular}

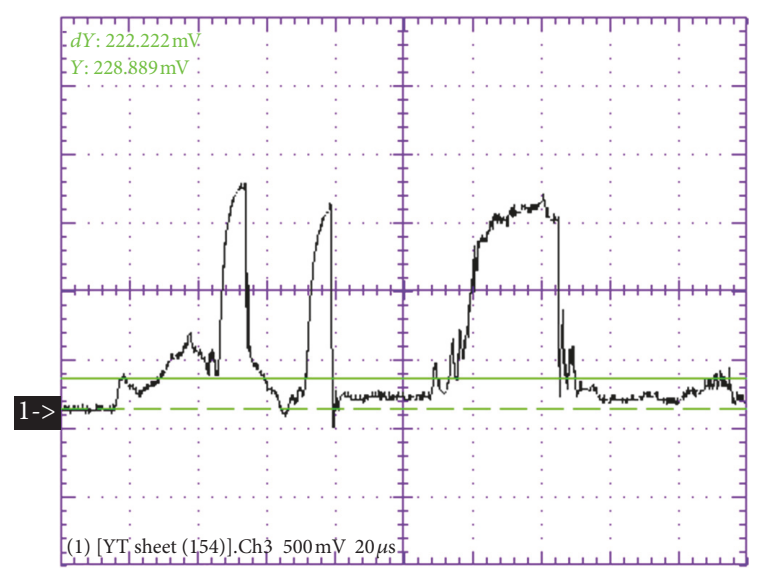

FIGURE 11: Typical pressure signals collected in the test.

Fitting the peak pressure of each measuring point, an equation of peak pressure is obtained:

$$
\begin{aligned}
& p_{m}=80.52995 \times e^{-X / 4.96494}+p_{0} \\
& p_{0}=1665.4 \times\left(Q^{1 / 3}\right)^{2.0608},
\end{aligned}
$$

where $p_{0}$ is the pressure of stable stage, $Q$ is explosive charge, $p_{m}$ is peak pressure, and $X$ is the distance from explosion center of perforation gun.

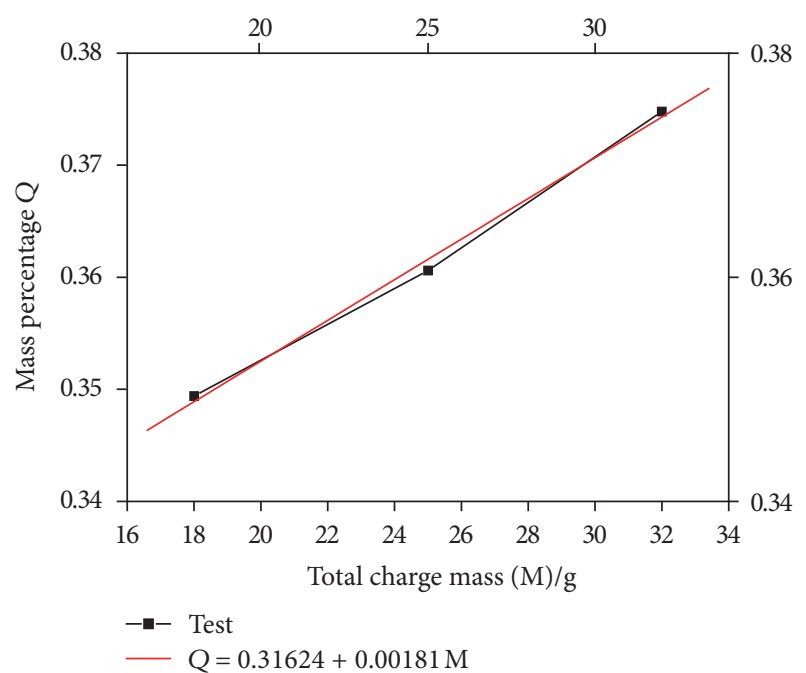

FIGURE 12: Relation curve between mass percentage and total charge.

According to the different types of perforating bullet, the engineering calculation method of the pressure field of downhole explosion can be established.

$$
p=p_{m} \times e^{-t / \theta}
$$

It is worth noting that the application of (17) should be restricted to a certain extent. The first one is that the explosion peak pressure and the pressure of steady stage depend only on the equivalent explosive mass of the downhole explosion of a 


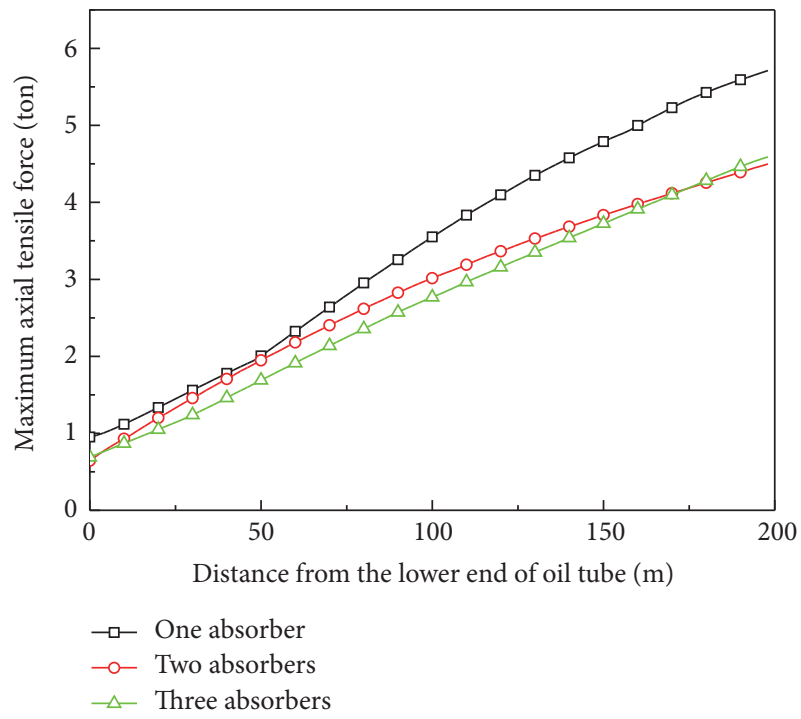

(a)

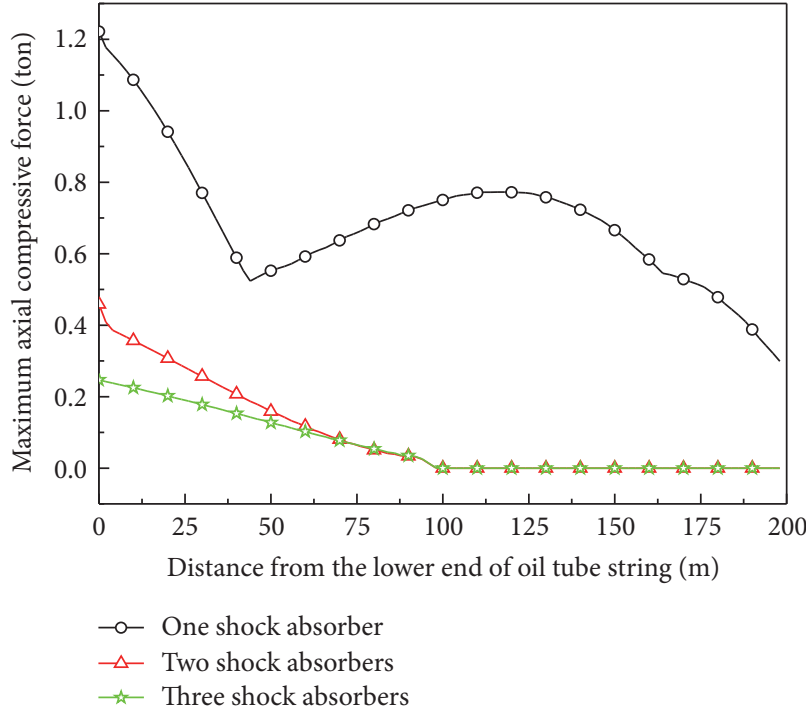

(b)

FIGURE 13: Effect of shock absorber on the maximum dynamic axial force in oil tube string: (a) tensile force; (b) compressive force.

single perforation bullet and they have no direct relation with the number of perforation bullets detonated simultaneously and the time interval of detonation. The second one is that (17) is only suitable for perforation bullet with RDX type charge. As other charges (TNT, HMX, and PYX) are used in perforation bullet, it is needed to modify the peak pressure and the pressure of stable stage.

\section{Dynamic Response Analysis of Downhole Tools}

A FORTRAN calculation code based on the dynamic model shown in Section 2 and the engineering calculation method of perforation pressure field shown in Section 3 are developed. Using the code, the dynamic response characteristics of the oil tube string and the packer in downhole perforation operation are investigated. The design, construction, and environmental parameters in calculation analysis are shown in Table 3.

4.1. Dynamic Response of Oil Tube and Packer. In the calculation code, an array is used to record the maximum axial tensile and compressive force in the oil tube string. It is noted that the upper end of the oil tube string (its position in the coordinate system is $200 \mathrm{~m}$ ) is connected to the packer. So, the axial force in the upper end of the tube string is equal to that acting on the packer.

Figures 13-15 show the distribution of the maximum dynamic axial force in oil tube string. It can be found that the axial tensile force decreases gradually along the well depth, with the maximum and minimum values appearing, respectively, at the upper and lower ends of the oil tube string. The distribution of maximum axial compressive force is just opposite to this phenomenon. The reason is that gravity is one of the main factors affecting axial forces.
TABLE 3: Basic perforation parameters.

\begin{tabular}{lc}
\hline Parameter & Value \\
\hline Liquid dynamic viscosity $(\mu)$ & $0.01 \mathrm{~Pa} \cdot \mathrm{s}$ \\
Yield strength of tubing material $(\sigma s)$ & $758 \mathrm{Mpa}$ \\
Outer diameter of oil tube & $88.9 \mathrm{~mm}$ \\
Inner diameter of oil tube & $76 \mathrm{~mm}$ \\
Length of oil tube string & $200 \mathrm{~m}$ \\
Element number of oil tube string & 100 \\
Elastic modulus of tubing material $(E)$ & $206 \mathrm{GPa}$ \\
Density of tubing material ( $\rho)$ & $7846 \mathrm{~kg} / \mathrm{m}^{3}$ \\
Total simulation time & $80 \mathrm{~s}$ \\
Time step & $0.001 \mathrm{~s}$ \\
Outer diameter of perforating gun & $73 \mathrm{~mm}$ \\
Inner diameter of perforating gun & $62 \mathrm{~mm}$ \\
Length of perforating gun & $3.3 \mathrm{~m}$ \\
Charge quantity of a perforating bullet & $16 \mathrm{~g}, 20 \mathrm{~g}, 32 \mathrm{~g}$, \\
Spring stiffness coefficient of shock & $48 \mathrm{~g}, 64 \mathrm{~g}, 128 \mathrm{~g}$ \\
absorber $(k)$ & $12 \sim 800 \mathrm{~N} / \mathrm{mm}$ \\
Damping coefficient of shock absorber $(c)$ & $15 \mathrm{~N} \cdot \mathrm{s} / \mathrm{mm}$ \\
Element number of perforating gun & 10 \\
Distance between the upper end of the & $0.2 \mathrm{~m}$ \\
perforating gun and the explosion center & \\
\hline
\end{tabular}

Figure 13 shows that, compared with one-shock absorber scheme, the two-shock-absorber scheme can significantly reduce the axial forces in the tube string. However, excessive arrangements of shock absorbers in the same position have little effect on reducing axial forces and force acting packer.

Figure 14(a) shows that, except for the upper and lower ends, the maximum dynamic tensile force in the same 


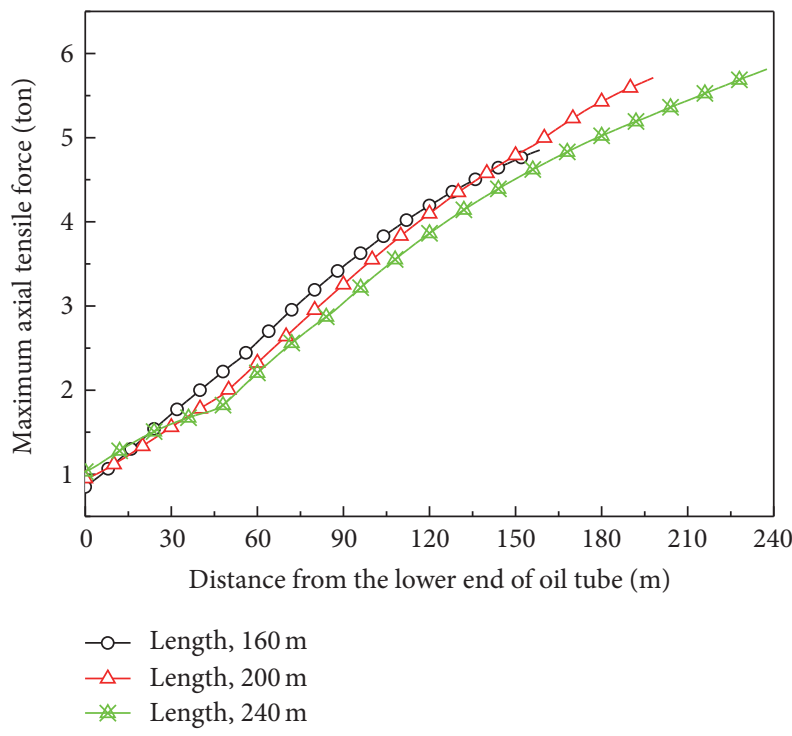

(a)

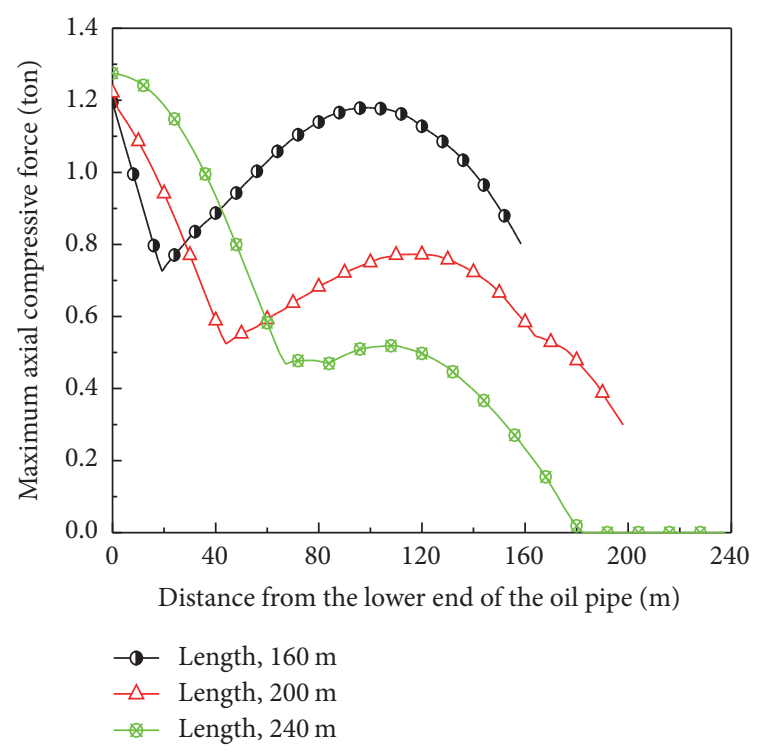

(b)

FIGURE 14: Effect of tube length on maximum dynamic axial force: (a) tensile force; (b) compressive force.

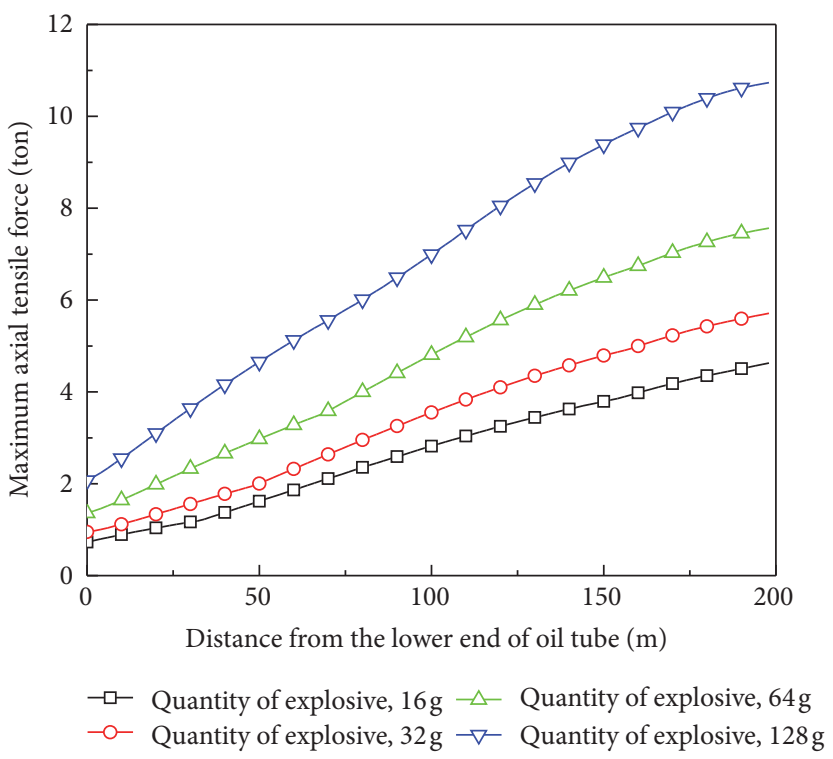

(a)

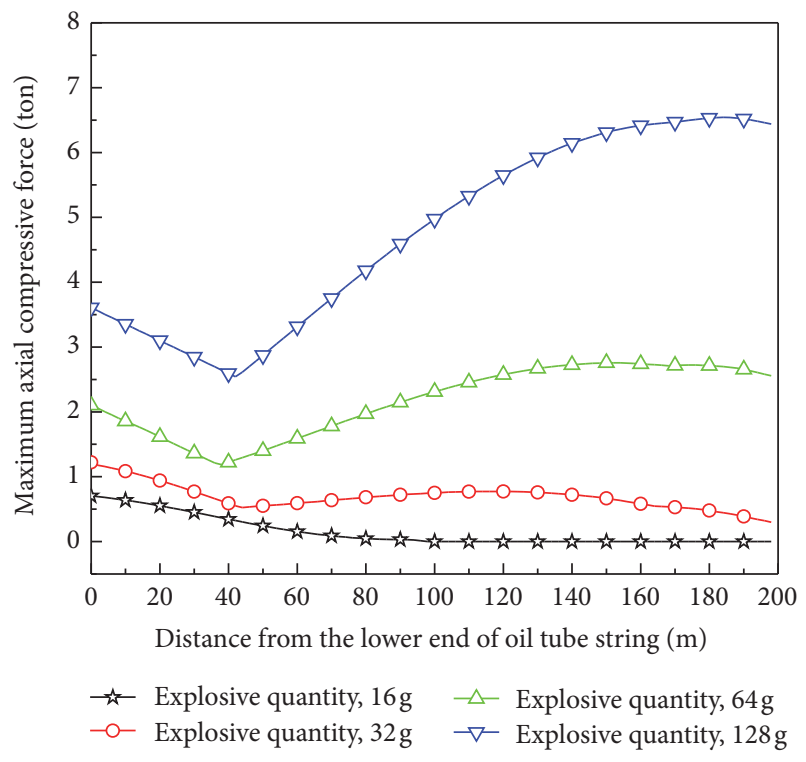

(b)

FIGURE 15: Effect of charge quantity on the maximum compressive force in oil tube string: (a) tensile force; (b) compressive force.

position decreases with increasing tube length. However, increasing tube length has no significant effect on the distribution of axial tensile force, whereas it is found in Figure 14(b) that the tube length has a significant effect on the distribution and magnitude of axial compressive force. As the length is relatively small, there are two extreme points appearing on the curve of axial compressive force. With the length increasing, the axial compressive force in the lower part of the oil tube string increases and that in the upper part decreases.

It is found in Figure 15 that the axial forces including tensile force and compressive force in oil tube string increase with the increase of charge quantity. The greater the distance from the lower end of oil tube string, the greater the increment. As the explosive charge changes, the axial tensile force shown in Figure 15(a) increases linearly with the distance. Figure 15(b) shows that with the charge quantity changing from low to high, the axial compressive force in the midupper part of tube string, compared with that in lower part of tube string, changes from weak to strong. So the explosive charge has significant effect on the axial force in oil tube, especially for mid-upper part of the tube string. For the case of large charge, the packer may fail, since the force acting on it may exceed the releasing force. 


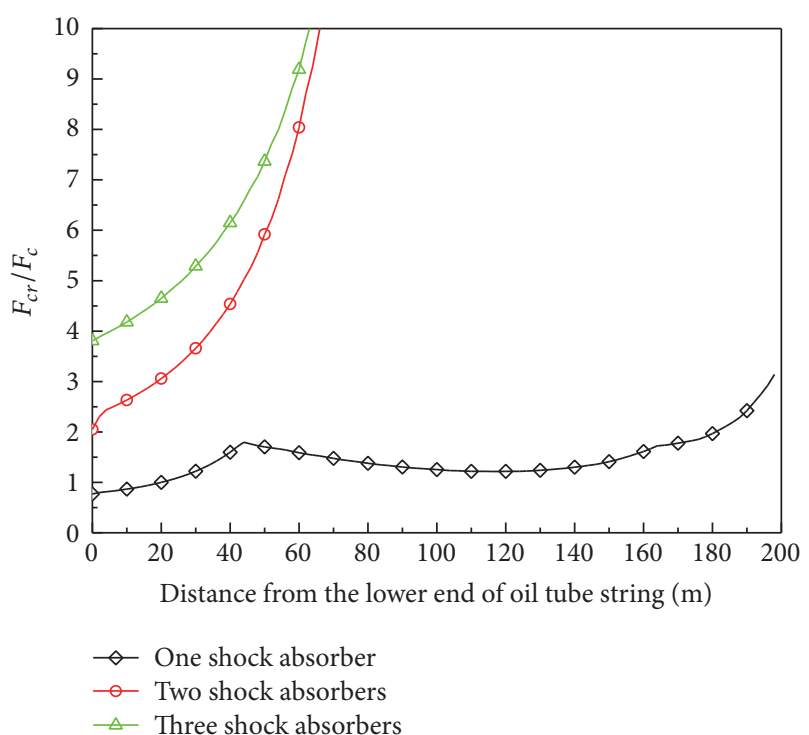

FIGURE 16: Effect of shock absorber on the buckling mechanical behavior oil tube string.

4.2. Buckling Mechanical Behavior of Oil Tube String. Helical buckling is a common failure form of tube string [24]. To investigate the perforation impact load on this mechanical behavior, an equation for calculating critical helical buckling load of tube string is used as follows [25]:

$$
F_{c r h}=5.55 \sqrt[3]{E I q^{2}}-\left(p_{o} S_{o}-p_{i} S_{i}\right)
$$

where $q\left(=\rho_{p} V g\right)$ is the effective line mass of oil tube, $E$ is elastic modulus, $\rho_{p}$ is density, $V$ is line volume per unit length, $I$ is inertia moment of cross section, $p_{i}$ and $p_{o}$ are pressures of the fluid in oil tube and annulus, and $S_{i}$ and $S_{o}$ are crosssectional areas corresponding to inner and outer diameters.

In order to distinguish the mechanical state of a tube string, here, a ratio $P_{r}\left(=F_{c r h} / F_{c, \text { max }}\right)$ is used, where $F_{c, \text { max }}$ is the maximum dynamic compressive force.

As $P_{r}<1$, helical buckling appears; otherwise, helical buckling does not appear.

Figure 16 shows that two-shock-absorber scheme is better than one-shock-absorber scheme in preventing helical buckling. Compared with two-shock-absorber scheme, threeshock-absorber scheme can only improve the capability of preventing helical buckling of the lower part of oil tube string; it has little effect on other parts. In fact, under the current conditions, one-shock-absorber scheme can meet the requirements of avoiding the helical buckling of the tube string. Figure 17 shows that with the tube length increasing the range of helical buckling in the lower part of tube string is expanded. However, in the other parts, the ability to resist buckling is enhanced. It is found in Figure 18 that with the charge of the perforating bullet increasing ratio $P_{r}$ decreases. Especially for the mid-upper part, this trend is more significant. It is indicated that the charge has a greater effect on the mid-upper part of the tube than the lower part of the string.

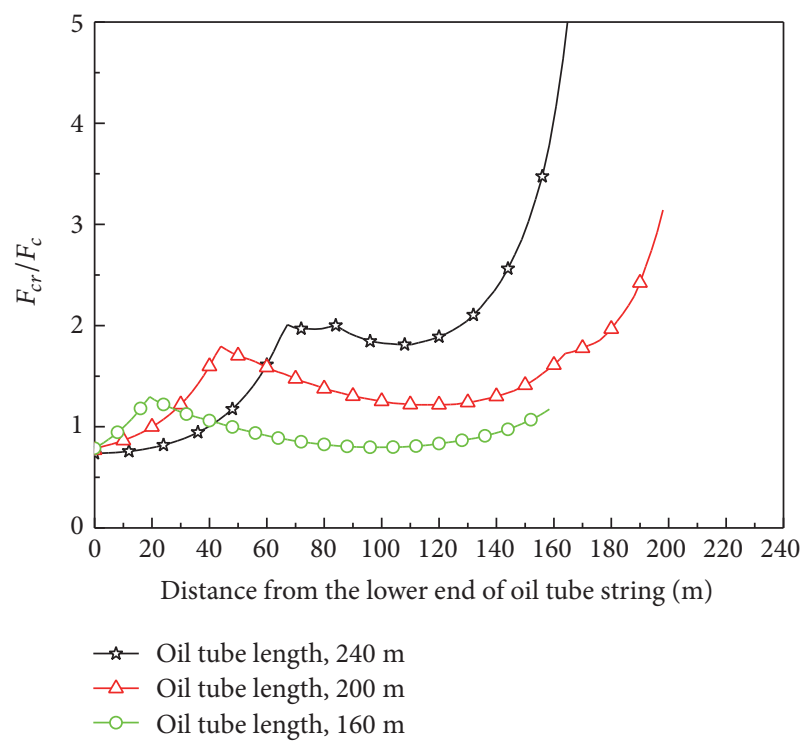

FIGURE 17: Effect of slenderness ratio of oil tube string on buckling mechanical behavior.

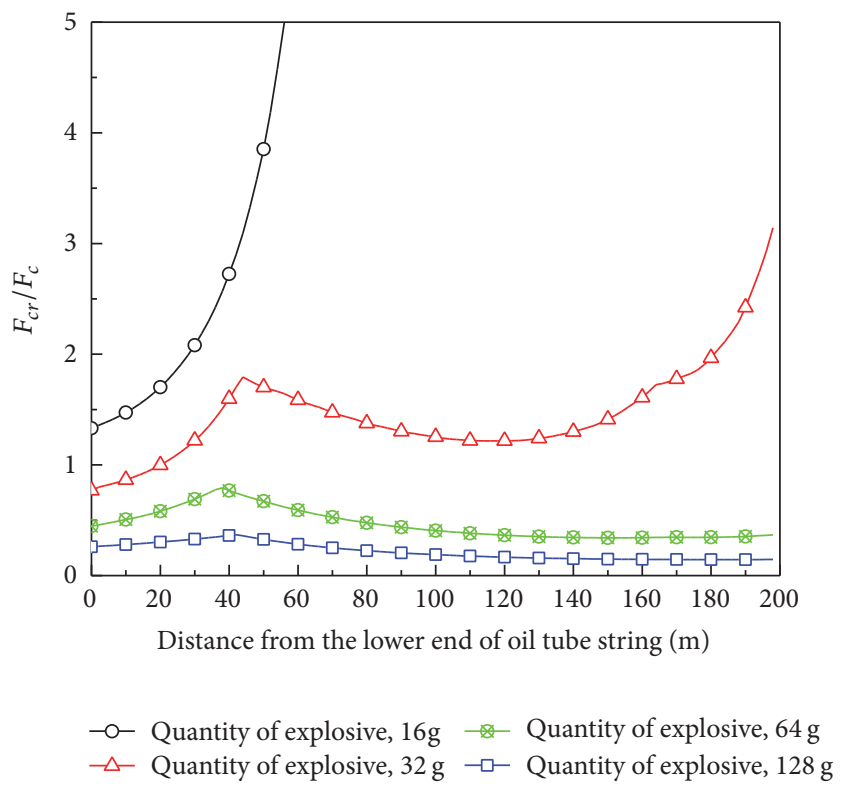

FIGURE 18: Effect of charge quantity on the buckling mechanical behavior of oil tube string.

\section{Discussion and Conclusion}

The effects of three important parameters, charge quantity, shock absorber number, and tube length, on the dynamic behavior of downhole tools have been investigated in detail. Based on the results described, the following conclusions can be drawn.

(1) The shock absorber between the perforating gun and the tube string can obviously improve the mechanical state of the oil tube and packer. However, it is not an economic option to set too many shock absorbers at the same location. 
(2) The charge has a significant effect on the mechanical state of tube string and packer. Especially for the mid-upper part of the tube, this effect is more evident. Thus, as the charge quantity is large, a subsection setting scheme of the shock absorber can be used in the mid-upper part of the tube string.

(3) The length increase of the tube string will reduce the stability of the lower part but will improve the stability of the mid-upper part. Thus, as the charge quantity is large, the mechanical state of oil tube string and packer can be improved by extending the length of the tube string below the packer.

\section{Conflicts of Interest}

The authors declare that there are no conflicts of interest regarding the publication of this paper.

\section{References}

[1] D. Bale, M. Ji, R. Satti, and J. Satti, "Gilliat. Advances in numerical modeling of downhole dynamics for perforated well completions," in Proceedings of the SPE Annual Caspian Technical Conference and Exhibition, pp. 12-14, 2014.

[2] Z. F. Li, C. Zhang, and G. Song, "Research advances and debates on tubular mechanics in oil and gas wells," Journal of Petroleum Science and Engineering, vol. 151, pp. 194-212, 2016.

[3] A. Lubinski and W. S. Althouse, "Helical Buckling of Tubing Sealed in Packers," Journal of Petroleum Technology, vol. 14, no. 06, pp. 655-670, 2013.

[4] A. Mcspadden, O. Coker, and G. Ruan, "Advanced casing design with finite-element model of effective dogleg severity, radial displacements, and bending loads," SPE Drilling and Completion, vol. 27, no. 3, pp. 436-448, 2012.

[5] X. H. Zhang, Y. Li, and Z. G. Dong, "Static and strength analysis of perforated string in side tracking horizontal well," Journal of Daqing Petroleum Institute, vol. 24, no. 2, pp. 57-59, 2000.

[6] Z. Li, Y. Cai, D. Li, and Y. Xu, "Mechanical analysis of pipe string in formation testing," Shiyou Xuebao/Acta Petrolei Sinica, vol. 32, no. 4, pp. 709-716, 2011.

[7] F. C. Guo, L. Tao, and X. B. Jia, "Mechanical model of horizontal drilling string and software development," China Petroleum Machinery, vol. 41, no. 7, pp. 28-32, 2013.

[8] D. Mu, M. Z. Zhou, and J. Song, "Application of the directional perforation technology under the condition of horizontal well screen completion," Chinese Journal of Solid Mechanics, vol. 33, pp. 314-318, 2013.

[9] G. C. Enss, R. Platz, and H. Hanselka, "Uncertainty in loading and control of an active column critical to buckling," Shock and Vibration, vol. 19, no. 5, pp. 929-937, 2012.

[10] X. Yang, F. Zhang, M. Li, and Y. Dou, "Analysis of strength safety of perforated string considering detonation parameters," Advanced Materials Research, vol. 634-638, no. 1, pp. 3573-3576, 2013.

[11] H. B. Chen, K. Tang, G. H. Ren, Z. K. Liao, and Y. Q. Ou, "String dynamic mechanics analysis on ultra-deep perforation," Well Logging Technology, vol. 34, no. 5, pp. 487-491, 2010.

[12] H. D. Yin, S. Y. Li, and J. J. Zhang, "Mechanics analysis of perforating combined well testing string and protection technology of downbole instrument," Oil drilling production technology, vol. 25, no. 3, pp. 61-62, 2003.
[13] H. W. Fan and K. Q. Li, "Perforation - Test Joint Pipe String and Process Optimization," Well Testing, vol. 22, no. 2, pp. 54-56, 2013.

[14] X. Lu, S. S. Wang, F. Ma, and H. F. Zhou, "Dynamic responses test of perforating string section under explosion impact," Science Technology and Engineering, vol. 14, no. 33, pp. 53-56, 2014.

[15] J. Liu, C. Huang, Y. F. He, R. B. Chen, and J. Z. Zhang, "Dynamic behavior study of perforated string system under impact load," Global Journal of Engineering Science and Research Management, vol. 2, no. 6, pp. 153-168, 2015.

[16] H. F. Zhou, Research on the dynamic response of the oil well perforating string to explosive impact load [M.S. thesis], Beijing Institute of Technology, 2014.

[17] Y. S. Teng, Dynamic simulation for perforating and string safety evaluation [M.S. thesis], China University of Petroleum, Beijing, China, 2014.

[18] K. Kang, F. Ma, H. Zhou, H. Wang, and S. Wang, "Study on dynamic numerical simulation of string damage rules in oil-gas well perforating job," Procedia Engineering, vol. 84, pp. 898-905, 2014.

[19] W.-B. Zhang, S. Xie, and Q. Lu, "Influencing analysis of perforation impact load on combined string of perforationacidification-test," Well Testing, vol. 25, no. 6, pp. 8-11, 2016.

[20] C. B. Li, Y. Liang, and Z. K. Lou, "Engineering calculation and application of perforation impact dynamic loads in oil and gas well," Advanced Materials Research, vol. 834-836, pp. 1308-1312, 2013.

[21] C. Huang, Study on dynamic behavior of deep water test tube under impact load [M.S. thesis], Southwest Petroleum University, 2015.

[22] L. Z. Cai, X. Zhao, S. F. Xue, and Z. Z. Yang, "Dynamic response analysis of str-ing structure during perforating process," Oil Field Equipment, vol. 44, no. 5, pp. 26-30, 2015.

[23] C. Tan, D. Gao, and J. Wang, "Stability of compression casingtubing-suck rod string in slant or curved well hole," Journal of the University of Petroleum, China, vol. 20, no. 6, pp. 21-23, 1996 (Chinese).

[24] W. Huang and W. Gao, "A local mechanical model of downhole tubular strings and its amendment on the integral model," in Proceedings of the IADC/SPE Asia Pacific Drilling Technology Conference, SPE-180613, Singapore, August 2016.

[25] M. Hajianmaleki and J. S. Daily, "Advances in critical buckling load assessment for tubulars inside wellbores," Journal of Petroleum Science and Engineering, vol. 116, pp. 136-144, 2014. 


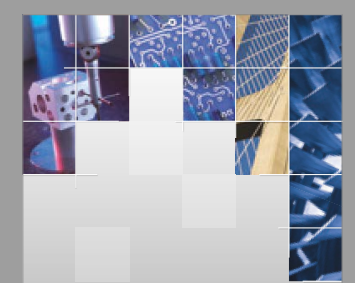

\section{Enfincering}
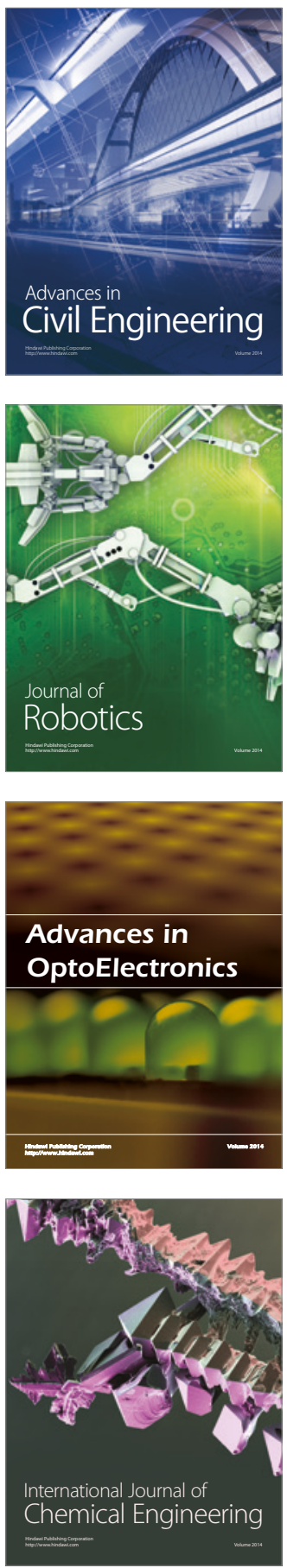

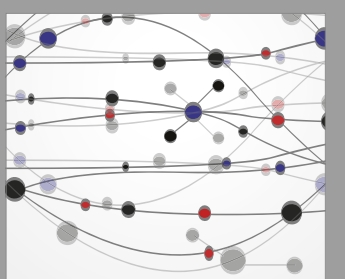

The Scientific World Journal

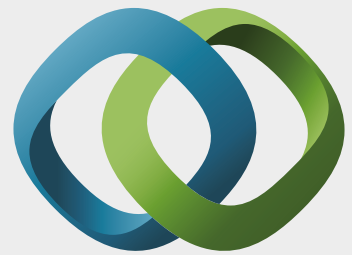

\section{Hindawi}

Submit your manuscripts at

https://www.hindawi.com
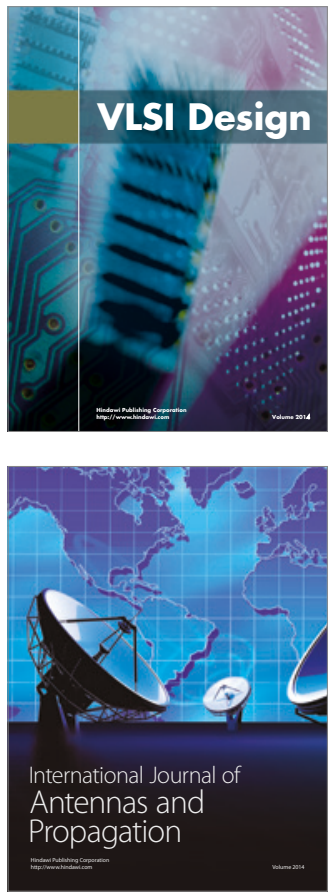

\section{Rotating}

Machinery
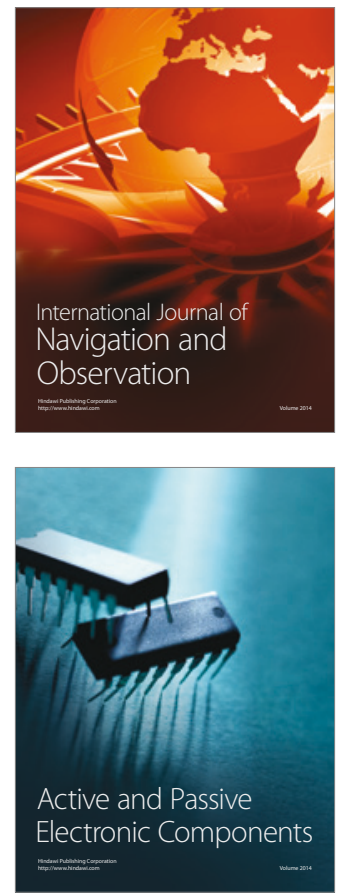
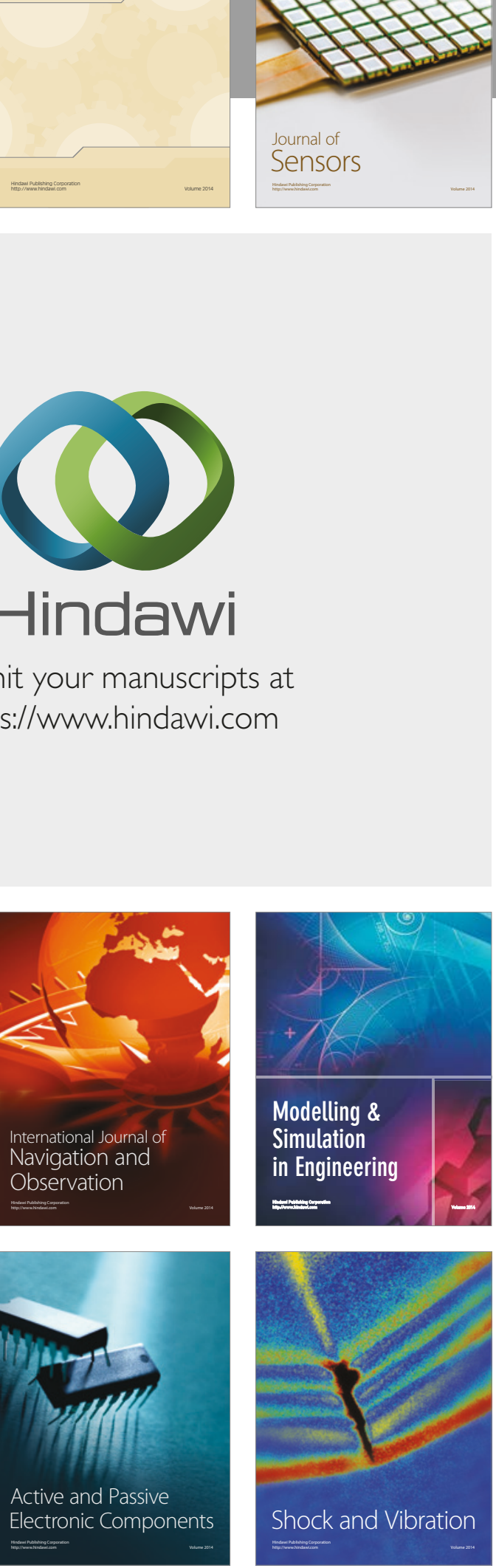
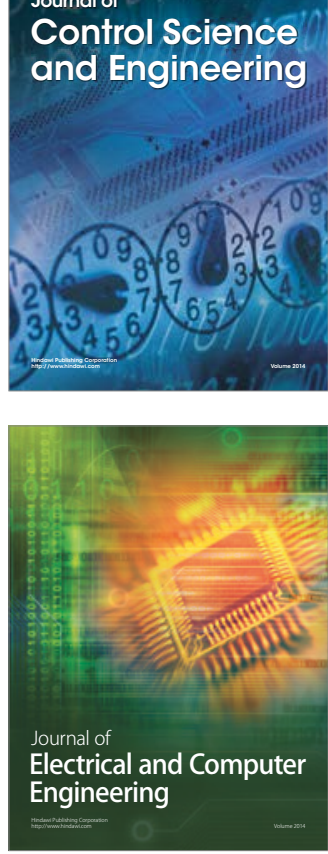

Distributed

Journal of

Control Science

and Engineering
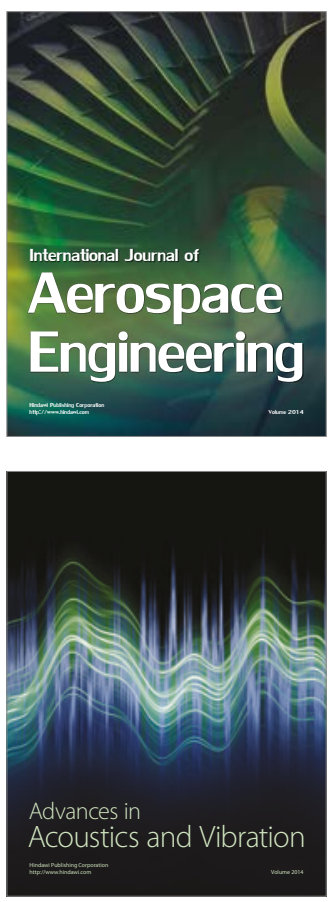

Sensor Networks 